\title{
Symptoms and visceral perception in severe functional and organic dyspepsia
}

\author{
H Mertz, S Fullerton, B Naliboff, E A Mayer
}

\begin{abstract}
Background-Hypersensitivity of gastric afferent pathways may play an aetiological role in symptoms of functional dyspepsia. Aims-To determine whether patients with severe organic dyspepsia (associated with tissue irritation/injury) and those with functional dyspepsia (no detectable tissue irritation) differ in their perception of gastric distension and whether this difference is reflected in differences in their gastrointestinal and psychological symptoms.
\end{abstract}

Methods-Perceptual thresholds, referral patterns, and gastrointestinal and psychological symptoms were compared in 23 patients with functional dyspepsia, 10 organic dyspeptics, and 15 healthy controls.

Results-Fifteen (65\%) functional dyspeptics and no organic dyspeptics had reduced perceptual thresholds for fullness, discomfort, or pain (odds ratio (OR) $19.56,95 \%$ confidence interval (CI) 1.95 to $476.09, p=0.0017)$. Either reduced perceptual thresholds or altered referral was found in $20(87 \%)$ functional dyspeptics and four (20\%) organic dyspeptics (OR $10.0,95 \%$ CI 1.34 to $89.54, p=0.014)$. During sham distension fullness, discomfort and pain were reported by healthy controls, organic dyspeptics, and functional dyspeptics. A sham response of pain but no other sensation was more frequent among functional dyspeptics (43\%) than healthy controls $(7 \%)$ (OR $10.77,95 \%$ CI 1.10 to $257.35, p=0.026)$. Gastrointestinal and psychological symptoms and gastric compliance were similar in the functional and organic groups.

CURE: Digestive

Disease Research

Center/Neuroenteric

Disease Program,

UCLA, Los Angeles,

California, USA

$S$ Fullerton

B Naliboff

E A Mayer

Department of

Medicine, Vanderbilt

University, Nashville,

Tennessee

H Mertz

Correspondence to:

Dr E A Mayer, Neuroenteric Disease Program, West LA

VA Medical Center, CURE

Bldg 115, Rm 223, 11301

Wilshire Blvd, Los Angeles,

California 90073, USA.

Accepted for publication 31 October 1997
Conclusions-Alterations in the perception of gastric distension distinguishes between functional and organic dyspepsia, while symptoms do not. A total of $87 \%$ of functional dyspeptics studied had evidence of altered visceral afferent function. In this study population, psychological abnormalities or changes in compliance did not explain the findings.

(Gut 1998;42:814-822)

Keywords: dyspepsia; hyperalgesia; visceral afferents

Despite the high prevalence of functional dyspepsia (FD) and its impact on the health care system, the aetiology and pathophysiology of the disorder remains poorly understood. Some estimates suggest that FD outnumbers peptic ulcer disease by 3:1 in prevalence, ${ }^{12}$ and may be present in up to $25 \%$ of Americans. ${ }^{3}$ Over $20 \%$ of affected individuals seek medical attention for their symptoms. FD related health care costs arise primarily from the fact that symptoms are frequently indistinguishable from organic causes of upper abdominal pain such as peptic ulcer disease, atypical gastrooesophageal reflux disease, or malignant disease of the upper abdomen. In the absence of detectable tissue injury or of a reliable biological marker, diagnosis is generally made by exclusion of organic disease.

Delayed gastric emptying, and more recently chronic gastritis secondary to Helicobacter pylori, have been implicated in the aetiology of FD, even though little experimental evidence supports a causal relationship of either finding with symptoms. In contrast, several pieces of evidence suggest that altered function of visceral afferent pathways may be responsible for both symptoms and delayed gastric emptying. ${ }^{4}$ FD symptoms are reported by approximately $40 \%$ of patients with irritable bowel syndrome (IBS), a syndrome thought to involve rectosigmoid hyperalgesia. ${ }^{5}$ Patients with FD or IBS have been found to have lower discomfort thresholds, and altered viscerosomatic referral areas during balloon distension of the stomach and rectum respectively. ${ }^{6-9}$

Even though perception of gastric balloon distension has been compared between patients with FD and normal subjects, the prevalence of hypersensitivity to gastric distension has not been well documented and the perceptual alteration has not been well characterised. Furthermore, it is not known whether patients with organic causes of dyspepsia (pain referred to the upper abdomen and/or nausea and associated with tissue injury or irritation) share the altered perception and referral of gastric distension.

In the current study, we sought to address the following questions: (1) what is the prevalence of altered perception of gastric distension in functional and organic dyspepsia? (2) Is there a correlation between symptoms of dyspepsia (organic or functional) with perception of gastric balloon distension? (3) Is hypersensitivity to gastric distension in patients with FD stimulus and sensation specific, similar to previous reports on hypersensitivity to rectal distension in IBS patients? ${ }^{67}$ We report that even though symptom prevalence between the two dyspepsia groups was similar, $87 \%$ of functional but only $20 \%$ of patients with organic dyspepsia showed evidence for altered perception of gastric distension. Thus, hypersensitivity of gastric afferent pathways to a peripheral mechanical stimulus cannot fully 
explain symptom generation in dyspepsia. Furthermore, chronic gastric or oesophageal irritation is not associated with gastric hyperalgesia. Part of these results have previously been reported in abstract form. ${ }^{10}$

\section{Methods \\ SUBJECTS}

All patients with dyspepsia (organic and functional) complained of pain, discomfort, or nausea referred to the upper abdomen (umbilical and above) occurring at least three times a week, and of at least three months duration. None of the subjects ingested more than $56 \mathrm{~g}$ of alcohol per day, took aspirin or non-steroidal anti-inflammatory agents, or displayed symptoms of overt psychopathology (DSM IV diagnoses of depression, anxiety disorder, or psychosis). All were seen by one of three physicians from the UCLA Center for Functional Bowel Disorders and Abdominal Pain. Patients were seen and evaluated at UCLA and at the CURE: Digestive Disease Research Center at the West LA Veterans Administration Hospital. Each patient completed a modified Talley Bowel Symptom Questionnaire, ${ }^{71}$ a psychological symptom checklist (SCL-90), ${ }^{12}$ and had a full history and physical examination by one of the three physicians; they were evaluated based on the physician's clinical assessment.

Functional dyspepsia patients

Twenty three patients between the ages of 20 and 74 (mean 46.7 years) were classified by their physicians as having FD. A diagnosis of FD was made based on a minimum history of three months of nausea, abdominal pain, and/or discomfort referred to the upper abdomen after exclusion of organic causes. Twenty one were seen at UCLA and two were seen at the CURE: Digestive Disease Research Center. Sixteen were female and seven were male. All had a normal upper endoscopy within three months of entry. To exclude atypical reflux, $57 \%$ had a 24 hour oesophageal $\mathrm{pH}$ study performed (all of which were normal) and all had failed either a four week trial of standard dose $\mathrm{H}_{2}$ blockers or omeprazole (40 $\mathrm{mg}$ /day). Patients with a history of either gastric surgery or vagotomy were excluded.

\section{Organic dyspepsia patients}

Ten patients with dyspeptic symptoms between the ages of 34 and 68 (mean age 52 years) underwent gastric sensory testing during their evaluation for abdominal pain. Sensory testing was ordered to diagnose or exclude functional dyspepsia. We excluded patients with specific organic diagnoses such as peptic ulcer disease. Four patients were female and six were male. Seven patients were seen at UCLA and three were seen at CURE. All patients had at least an oesophagogastroduodenoscopy during their evaluation. Organic pathology was detertnined by endoscopy in four patients (two duodenal ulcers, one gastric ulcer, and one oesophagitis), by 24 hour oesophageal $\mathrm{pH}$ monitoring in three (all gastro-oesophageal reflux), by computed tomography (CT) scan in two (one hepatoma and one dense adhesions with gastric entrapment), and blood tests in one (porphyria). The organic diseases were thought to explain the dyspeptic symptoms in all 10 subjects based on clinical impression. In three with ulcers, symptoms resolved with acid suppression. In one with oesophagitis and three with abnormal oesophageal $\mathrm{pH}$ studies, symptoms resolved with proton pump inhibitors. One patient had gastric entrapment with severe distortion of her stomach by CT scan and endoscopy. These abnormalities were thought to explain her early satiety and dyspepsia. She did not undergo surgery and the cause of the symptoms was therefore not definitively confirmed. One patient had a hepatoma and his symptoms progressed as the tumour enlarged. In one patient, acute intermittent porphyria by signs, symptoms, and urine testing was thought to explain her symptoms.

In all cases (functional and organic) a diagnosis had not been established at the time of gastric sensory testing. The performing technician and the interpreting physician were blinded to the final diagnosis.

\section{Healthy volunteers}

Fifteen healthy subjects (14 men, one woman) between the ages of 21 and 55 (mean age 35) were recruited by advertisement. All had gastric sensory testing at CURE. None had any history of chronic illness or evidence for chronic abdominal pain or discomfort. Each subject was screened with a history and physical examination to ensure no medical problems were present. Normal gastric distension volume thresholds for sensations of fullness, discomfort, and pain were determined by taking the mean of the 15 healthy controls ( $90 \%$ confidence interval). The study protocol was approved by the West Los Angeles Veterans Administration Human Subjects Committee.

EQUIPMENT

Displacement device

A computer driven programmable volume displacement device (Synectics Visceral Stimulator, Synectics, Stockholm) was used in these studies to distend the stomach. ${ }^{13}$ The device can deliver volume ramps or phasic pressure steps while simultaneously monitoring pressure and volume at a sampling rate of one per second. Pressure is monitored within the inflation device. The software compensated for the compressibility of air using the Ideal Gas Law. This correction was validated by clamping the air line of the device during ramp distension. Under these circumstances, no volume displacement and a straight increase in pressure was observed. The threshold of perceived sensations (fullness, discomfort, pain) was recorded on the pressure volume curve when the subject pushed one of three buttons on a marker device. Balloon deflation was immediately triggered either when the subject indicated pain, or when the device reached the pressure limit of $60 \mathrm{~mm} \mathrm{Hg}$, or a volume of $1600 \mathrm{ml}$. The response characteristics of the device have previously been reported in detail. ${ }^{14}$ 
Distending balloon

A latex balloon was used as the distending balloon. The condom was trimmed to a length of $10 \mathrm{~cm}$ and tied to the distal end of a standard nasogastric tube. Distension outside of the subject resulted in a spherical balloon shape with a diameter of $14 \mathrm{~cm}$ when fully inflated to $1600 \mathrm{ml}$. The balloon was tested before and after experiments to ensure there was no leak. Following three preinflations during which compliance increased by up to $10 \%$, balloon compliance remained stable during up to 20 inflations. The balloon compliance was determined before the distension by an inflation outside of the patient, then automatically subtracted by the barostat from the patient compliance curve. Balloon compliance was the same before and after use.

\section{Procedure}

The posterior pharynx was anaesthetised with topical anaesthetic spray (20\% benzocaine) prior to balloon insertion. The deflated balloon and nasogastric tube were passed orally into the stomach with less than $50 \mathrm{ml}$ of swallowed water and a small amount of surgilube jelly. The balloon was advanced to $50 \mathrm{~cm}$ from the incisors and inflated to a volume of $300 \mathrm{ml}$. Gentle traction was used to position the balloon in the gastric fundus. The tube was taped to prevent migration and the balloon was deflated. $x$ Ray images of the balloon were obtained for three patients not enrolled in this study to evaluate the geometry of the balloon during distension in vivo. In all cases the balloon expanded to fill the fundus and antrum with a gourd like shape early during inflation and continued to expand equally in all directions preserving the same shape from volumes of $150 \mathrm{ml}$ to $1600 \mathrm{ml}$.

\section{Testing environment}

Subjects reclined on a padded table and wore headphones throughout the test to block any auditory cues arising from the noise of the volume displacement device. The examiner was always present, but did not interact with the subjects after explanation of the protocol at the beginning of each test. Experiments were performed between 8 am and 11 am after a six to 12 hour fast. There were two identical testing sites, one at UCLA and one at CURE. The technicians and interpreting physician were the same at each site.

STUDY PROTOCOL

Gastric distension was performed as previously reported $^{14}$ using a slow volume ramp (all subjects) and predetermined irregularly ascending stepwise isobaric pressure changes (phasic distension; FD and controls). Patients were not informed about the existence of "sham" distension runs. The phasic and sham protocols were included to estimate the contribution of response bias to observed perception thresholds.

Ramp distension protocol

Intragastric distending volume was administered at a constant rate of $200 \mathrm{ml} / \mathrm{min}$ to progressively distend the stomach. This rate was based on preliminary studies aimed at determining physiological rates of gastric distension. This rate is well below the maximal rate of gastric distension during rapid fluid ingestion, which we determined by asking 12 healthy volunteers to drink $400 \mathrm{ml}$ of tap water $\left(22^{\circ} \mathrm{C}\right)$ at a rapid steady pace. The rate of water ingestion ranged between 311 and $685 \mathrm{ml} / \mathrm{min}$ with a mean value (SEM) of $472(35) \mathrm{ml} / \mathrm{min}$.

One "practice" inflation was performed initially to make the patient familiar with the distension protocol and the response device. Three ramp inflations were then performed, each ending if pain was reported, pressure reached $60 \mathrm{~mm} \mathrm{Hg}$, or volume reached 1600 $\mathrm{ml}$.

\section{Sham distension}

Two sham distortions were performed randomly between the ramp distortions. Sham distortions were only performed after a subject had experienced a true ramp distension of the stomach. During the sham distension, the inflating catheter was disconnected from the barostat pump without the patient's knowledge. Since subjects wore headphones, and could not see the volume displacement device, they could not use auditory or visual cues to estimate the level of distension. Patients were instructed to record symptoms during sham distortions just as they did during actual distortions.

\section{Phasic distension protocol}

In order to determine whether lowered thresholds previously reported in patients with FD were related to response bias rather than afferent hypersensitivity, we included the following protocol in the assessment of six patients with FD and 14 healthy controls. Phasic distortions are defined here as rapid isobaric distortions. Rapid isobaric distortions of 60 seconds duration were administered at a volume rate of 960 $\mathrm{ml} /$ minute in a predetermined irregularly ascending sequence as follows: $16,18,8,12$, 20,26, 22 and $28 \mathrm{~mm} \mathrm{Hg}$. To account for pressure due to balloon resistance, balloon pressures from 2 to $10 \mathrm{~mm} \mathrm{Hg}$ (depending on balloon volume) were subtracted from these pressures to yield net gastric pressure. Between isobaric steps, a baseline pressure of $4 \mathrm{~mm} \mathrm{Hg}$ was maintained for 60 seconds. The study was terminated if pain was reported, or $60 \mathrm{~mm} \mathrm{Hg}$ or $1600 \mathrm{ml}$ was reached. The phasic protocol was performed once, after the first two ramp distensions. Phasic distension had no effect on thresholds observed during subsequent ramp distension.

\section{Perceptual thresholds and verbal descriptors}

Subjects recorded symptoms during the distensions by pressing one of three buttons on a response device (1, fullness; 2 , discomfort; 3 , pain). At the initial history and physical examination, subjects drew a diagram of the location and size of their presenting symptom on a body map, and during the sensory testing they indicated the cutaneous sensory referral area of the distending stimulus (fig 1). 


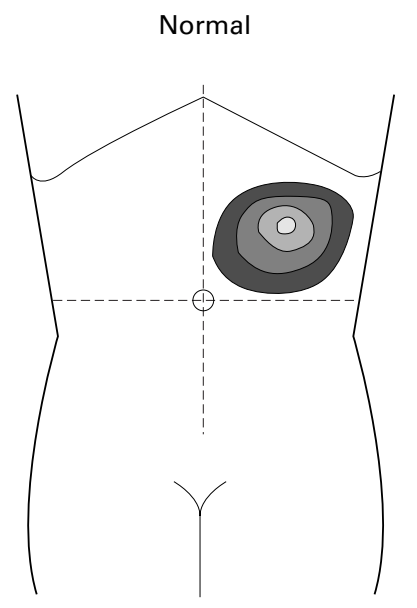

Functional dyspepsia

Figure 1 Viscerosomatic referral during gastric distension. A typical referral pattern from a healthy control subject (also typical of the organic dyspeptics) and the corresponding pattern from a patient with $F D$.

Perceptual thresholds were measured in terms of pressure and volume. Body surface area (BSA) in square metres was calculated for each subject and was used to normalise sensory threshold volumes for variations in body (and presumably gastric) size, by dividing thresholds (ml) by BSA $\left(\mathrm{m}^{2}\right)$. Perceptual thresholds during ramp inflation for each sensation (fullness, discomfort, and pain) were determined by averaging the results from the three ramps (not including the practice ramp). For phasic distension, the thresholds were determined from one sequence.

Gastric compliance

Pressure and volume in the gastric distension balloon were continuously recorded by the barostat during distension, allowing us to determine pressure-volume relationships. Pressures were corrected for balloon compliance by subtracting balloon pressures at each volume. Baseline intragastric pressures prior to any air inflation were calibrated as $0 \mathrm{~mm} \mathrm{Hg}$. Compliance was defined as the ratio of change in intragastric volume and pressure from a baseline of zero. Gastric compliance between patient groups was compared during ramp and during isobaric distension. During volume ramp distension, respective intragastric pressures were

Table 1 Comparison of symptoms and gastric sensory testing in patients with functional dyspepsia, organic dyspepsia and controls

\begin{tabular}{llll}
\hline & $\begin{array}{l}\text { Functional dyspepsia } \\
(n=23)\end{array}$ & $\begin{array}{l}\text { Organic dyspepsia } \\
(n=10)\end{array}$ & Controls $(n=15)$ \\
\hline Effect on life $(1-10) \ddagger$ & $6.2(1)$ & $6.9(1)$ & $\mathrm{NA}$ \\
Pain severity $(1-4) \ddagger$ & $3.1(1)$ & $3.1(1)$ & $\mathrm{NA}$ \\
Exacerbated by food & $6 / 17(35 \%)$ & $3 / 7(43 \%)$ & $\mathrm{NA}$ \\
Early saity & $15 / 19(79 \%)$ & $4 / 7(57 \%)$ & $\mathrm{NA}$ \\
Weight loss $(>3.18 \mathrm{~kg})$ & $10 / 19(55 \%)$ & $2 / 7(37 \%)$ & $\mathrm{NA}$ \\
Nausea a primary complaint & $11 / 18(61 \%) \dagger$ & $0 / 8(0 \%)$ & $\mathrm{NA}$ \\
Nausea weekly & $11 / 20(55 \%)$ & $2 / 7(28 \%)$ & $\mathrm{NA}$ \\
Vomiting weekly & $7 / 21(33 \%)$ & $3(37 \%)$ & $\mathrm{NA}$ \\
GORD six weekly & $6 / 20(30 \%)$ & $1 / 6(17 \%)$ & $\mathrm{NA}$ \\
Insomnia & $12 / 17(71 \%)$ & $5 / 8(62 \%)$ & $\mathrm{NA}$ \\
Hypersensitive & $16 / 23(65 \%)^{\star \star \star}$ & $0 / 10(0 \%)$ & $0(0 \%)$ \\
Normal referral & $12 / 23(52 \%)^{\star \star}$ & $2 / 10(20 \%)$ & $0(0 \%)$ \\
Either & $20 / 23(87 \%)^{\star \star \star}$ & $2 / 10(20 \%)$ & $0(0 \%)$ \\
Symptoms reproduced by & & & \\
$\quad$ balloon distension & $13 / 19(68 \%)^{\star}$ & $3 / 10(30 \%)$ & $\mathrm{NA}$ \\
\hline
\end{tabular}

${ }^{\star} \mathrm{p}<0.05 v$ organic dyspepsia; ${ }^{\star \star} \mathrm{p}<0.01 v$ controls; ${ }^{\star \star \star}{ }^{\star \star} \mathrm{p}<0.001 v$ organic dyspepsia and controls $\mathrm{tp}<0.01 v$ organic dyspepsia.

$\ddagger$ Results expressed as mean (SEM).

GORD, gastro-oesophageal reflux disease. compared at $50 \mathrm{ml}$ intervals. During isobaric distension, respective intragastric volumes were compared for each distension pressure.

\section{Assessment of psychological symptom severity} Patients completed the SCL-90 psychological symptom questionnaire at the time of their initial clinic visit. Nine symptoms thought to be indicative of non-ulcer dyspepsia (NUD) and all eight of the major psychological symptom scales were selected for analysis prior to review of the data. No other symptom data were included in the analysis. Nineteen of 23 patients with FD (83\%) and eight of 10 organic patients $(80 \%)$ completed the questionnaire. The SCL-90 yields $\mathrm{T}$ scores based on a non-psychiatric normative sample for symptom scales of anxiety, depression, somatisation, obsessive compulsive traits, interpersonal sensitivity, hostility, phobic traits, and paranoia. Clinically significant levels of psychology symptoms are defined as a $\mathrm{T}$ score above 63 (normative mean 50, SD 10). ${ }^{12}$

\section{Statistical methods}

Group difference for continuous variables was assessed using Student's $t$ test. Results are expressed as mean (SEM). Associations for categorical variables were measured using Fisher's exact test. Pearson product moment correlations were obtained for comparisons of psychological symptoms (SCL-90 severity scores) and age with perceptual thresholds. For the 17 gastrointestinal and psychological symptoms evaluated, results are presented without adjustment for multiple comparisons. Actual $\mathrm{p}$ values are reported.

\section{Results}

SYMPTOMS

Patient symptoms were assessed with a bowel symptom questionnaire. Patients with FD commonly had symptoms of nausea at least weekly $(55 \%)$, nausea as a primary complaint $(61 \%)$, and early satiety $(79 \%)$, and reported weight loss of more than $3.18 \mathrm{~kg}$ in the last year (55\%). Symptoms of gastro-oesophageal reflux (30\%), vomiting $(33 \%)$, and exacerbation of symptoms by food (35\%) were less common in the patients with FD. Compared with the organic patients, only nausea as a primary complaint was significantly more common in FD ( $p=0.0074)$ (table 1). Pain was rated on average as severe (rating of 3 on a four point scale) by both FD and organic patients. The negative effect of the condition on private and professional life was rated as 7 out of a possible 10 (on a visual analogue scale, where 10 is most severe and 0 is insignificant) for both patient groups.

Patients with FD who reported nausea at least weekly $(n=9)$ were more likely than those with less frequent nausea $(n=8)$ to have an abnormal level of psychological symptoms. Those with more frequent nausea were more depressed ( $6 / 9$ versus $1 / 8)$, tended to be more somatically focused (6/9 versus $2 / 8)$, and tended to be more hostile ( $5 / 9$ versus $1 / 8$ ).

Psychological symptoms were present in approximately $50 \%$ of patients, and were 


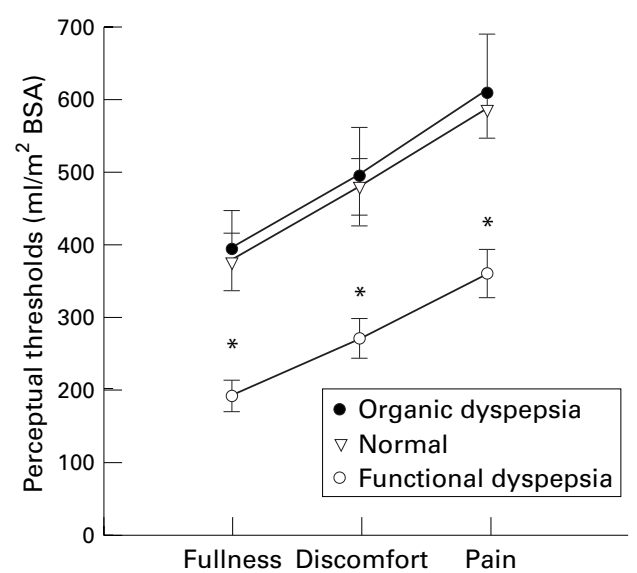

Figure 2 Perceptual response to gastric distension (ramp). ${ }^{*} p<0.01$ for $F D$ versus organic dyspeptics and healthy controls.

equally common in the FD and organic dyspeptics. Clinically significant psychological symptoms were seen in $11 / 19$ patients with FD and $5 / 8$ organic patients. For anxiety and depression, 9/19 patients with FD and 2/8 organic patients had significant levels of symptoms. For somatisation, obsessive compulsive trait, interpersonal sensitivity, hostility, phobic trait, and paranoia, 10/19 patients with FD and $5 / 8$ organic patients had significant levels of symptoms. When mean values of scores for each psychological symptom were compared, there were no significant differences between the functional and the organic dyspeptics for any symptom category.

PERCEPTUAL THRESHOLDS

Ramp distension

Patients with FD were notably more sensitive (lower thresholds) to gastric distension than normal controls or patients with organic causes of dyspepsia. Volume thresholds for fullness, discomfort, and pain (reported in that order by $46 / 48$ subjects) in response to balloon distension were $40-50 \%$ lower in patients with FD than in patients with organic causes of dyspepsia or healthy volunteers $(p<0.01$ for each of six

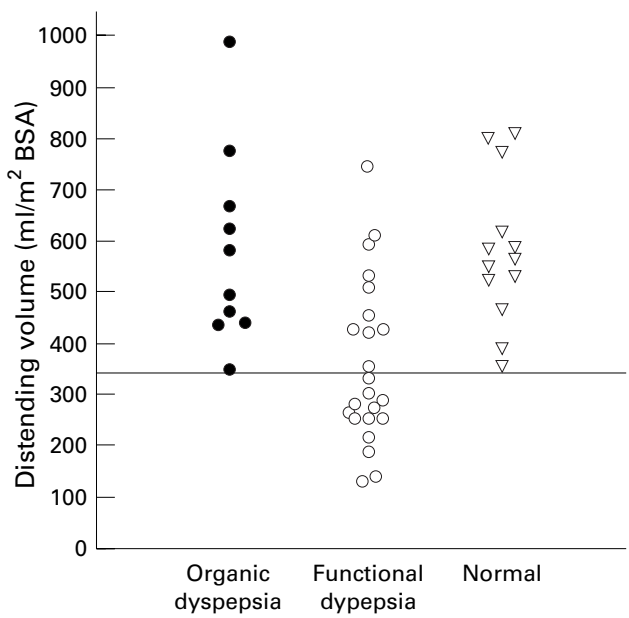

Figure 3 Pain thresholds during gastric distension (ramp). The cut off line for a normal pain threshold was selected to include all the healthy controls. This cut off was the mean -1.6 SD (90th percentile) (2/15 healthy controls did not report pain up to gastric volumes of $1500 \mathrm{ml}$ ). comparisons) (fig 2). Thresholds for patients with organic dyspepsia and healthy controls were similar. No patients with organic dyspepsia had hypersensitivity to distension (using normal values determined from the 15 healthy volunteers). Fifteen of 23 patients with FD $(65 \%)$ had a reduced threshold for one of the three sensations. Thresholds for fullness, discomfort, and pain were reduced in 12,13 , and 13 patients with FD respectively (fig 3). Although there was a greater portion of women in the FD group, there was no significant difference in sensory thresholds between males and females. In this study, the findings of reduced sensory thresholds to gastric ramp distension were specific for FD. None of the organic dyspeptics or the healthy volunteers had reduced thresholds, but $65 \%$ of the patients with FD showed hypersensitivity.

Pressure thresholds for fullness $(\mathrm{F})$, discomfort (D), and pain (P) with ramp distension were similar in FD (F: $12.2(0.9)$; D: 13.3 (0.7); P: 14.7 (0.7)), organic dyspeptics (F: 14.5 (1.3); D: 15.0 (1.3); P: 16.1 (1.4)), and normal controls (F: 12.5 (0.7); D: 13.6 (0.5); P: $14.6(0.5))$. The pressure for each successive sensation was similar to the pressure for the previous one since the sensations tended to occur in the flat plateau portion of the gastric pressure volume curve (fig 4).

\section{Phasic distension}

In order to rule out possible effects of response bias on perception thresholds, we also evaluated thresholds during a series of predetermined irregularly ascending isobaric (phasic) distension steps in six patients with FD and 14 healthy volunteers. Patients with FD were more sensitive to rapid isobaric distension than normal control subjects for discomfort and pain, but not fullness. The volume threshold for fullness in FD was 219 (48) versus 292 (48) in normal controls $(\mathrm{p}=0.37)$. For discomfort the threshold in FD was 266 (52) versus 482 (53) in controls $(\mathrm{p}=0.02)$. For pain the threshold in FD was 387 (72) versus $665(70)$ in controls $(p=0.01)$. The sensory pressure thresholds were similar for FD (F: 12.1 (1.8); D: 15.6 (1.5); P: 17.6 (1.3)) and normal controls (F: 13.6 (1.3); D: 17.5 (0.7); P: 18.4 (0.7)).

In controls, perceptual thresholds obtained during ramp and phasic distension showed a weak, but significant correlation for all three sensations ( $\mathrm{F}: r^{2}=0.80, \mathrm{p}<0.01 ; \mathrm{D}: r^{2}=0.43$, $\mathrm{p}<0.02$, P: $\left.r^{2}=0.49, \mathrm{p}<0.05\right)$, while in patients with FD such a correlation was only observed for discomfort $\left(r^{2}=0.68, \mathrm{p}<0.05\right)$.

Correlation of perception thresholds with clinical parameters

We evaluated the effect of age, patient sex, and psychological symptoms on sensitivity to gastric distension. Perceptual thresholds for all sensations during ramp distension were influenced by patient age, with older patients less sensitive (higher thresholds). This effect was seen in the FD group for fullness, discomfort, and pain. For healthy controls this reached significance for pain. For organic patients this trend did not reach significance (table 2). 
Table 2 Effect of patient age on thresholds during ramp distension

\begin{tabular}{llll}
\hline & Fullness & Discomfort & Pain \\
\hline Functional dyspepsia & $3.3(0.29)^{\star}$ & $4.1(0.3)^{\star}$ & $4.6(0.26)^{\star}$ \\
Organic dyspepsia & $9.1(0.32)$ & $8.8(0.19)$ & $0.02(0.16)$ \\
Control & $6.6(0.24)^{\star}$ & $7.0(0.22)$ & $0.04(0.3)^{\star}$ \\
All & $3.1(0.09)^{\star}$ & $3.2(0.07)$ & $3.7(0.08)$ \\
\hline
\end{tabular}

Results are slopes of lines $(y=m x+b)$ describing correlations of ramp perpetual thresholds to patient age, where $x=$ age and $y=$ threshold/BSA. $T^{2}$ values for correlations are in parentheses. ${ }^{\star} \mathrm{p}<0.05$.

There was no correlation between age and phasic perceptual thresholds. Male and female patients had similar sensitivities to distension. There was no relation between psychological symptom severity (by SCL-90) and perceptual thresholds. There was no difference in clinical symptoms between normal and low threshold patients with FD.

GASTRIC PRESSURE VOLUME CURVE

The gastric pressure in response to progressive volume ramp distension was a characteristic hyperbolic shape, with an early steep phase followed by a plateau (fig 4 ). In no patient did the maximum plateau pressure exceed $22 \mathrm{~mm} \mathrm{Hg}$. Sensations of fullness, discomfort, and pain were characteristically experienced in the plateau portion of the compliance curve. There was no difference in the pressure volume curves of patients with FD, organic dyspeptics, and control subjects. Comparisons above 800 $\mathrm{ml}$ could not be made because few patients with FD tolerated distension beyond that volume (fig 4A).

During isobaric distension, gastric expansion was initially very rapid, followed by a slower accommodation. At pressures above 13 $\mathrm{mm} \mathrm{Hg}$ a volume plateau was not typically reached before the subject experienced pain $(86 \%), 1600 \mathrm{ml}$ was reached, or the end of the 60 second isobaric step was reached. Peak volumes at each isobaric step plotted against pressure showed the same hyperbolic shape as ramp distension (fig 4B). There was no difference in volume responses to isobaric distension between patients with FD and healthy controls.
ANATOMICAL SITE OF SENSATIONS

Subjects were asked to draw on a body map the area where they perceived the sensations of fullness, discomfort, and pain during gastric ramp distension (fig 1). In normal subjects this area always (15/15) centred around the epigastrium, left upper quadrant, or periumbilical region. With progressive distension, the areas of sensation were typically drawn as concentric circles, with the more aversive sensations (pain $>$ discomfort $>$ fullness) experienced at larger volumes and drawn as larger circles. The referral of each sensation was always in continuity (overlapping) with the previous, less aversive sensation. Twelve of the 23 patients with FD experienced one or more of the sensations in an aberrant location. These sites included the back (two), right upper quadrant (six), lower abdomen (six), and pelvis (one). The aberrant referral area was often discontinuous with the other sensation areas $(10 / 23$ or $43 \%)$. Eight of the 10 organic disease patients had normal referral areas. Of the two patients with aberrant referral, retrosternal referral was seen in one with reflux disease, and referral to the back was seen in another with painful duodenal ulcer. In both patients the reported referral area reported during distension was similar to the site identified previously by the patient as location of their typical chronic pain. Typical pain was more likely to be provoked with gastric balloon distension in patients with FD (68\%) than in organic patients $(30 \%)(p=0.047)$.

If abnormal sensation is defined as either aberrant referral or hypersensitivity to gastric distension, 20/23 (87\%) patients with FD were abnormal, compared with only $2 / 10(20 \%)$ organic patients $(p=0.0002)$, and $0 / 15$ normal volunteers $(p<0.0001)$. Sensitivity of abnormal sensation for FD is $87 \%$ and specificity is $80 \%$, when compared with an appropriate disease control.

\section{SHAM DISTENSION}

Sham balloon distension was performed randomly during the inflation protocol, by
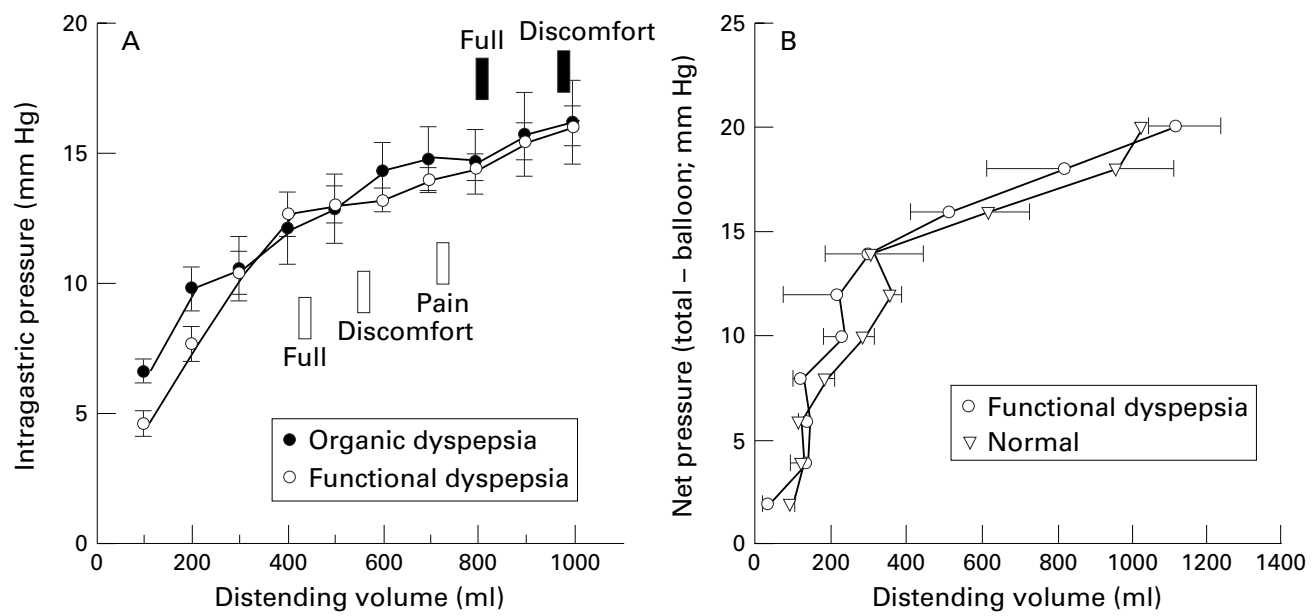

Figure 4 Gastric compliance. (A) Ramp distension. Gastric compliance (dV/dP) at each volume was the same in functional dyspeptics, organic dyspeptics, and healthy control subjects (not shown for clarity). Gastric perceptual thresholds in relation to the pressure/volume curve are indicated for functional dyspeptics (open boxes) and organic dyspeptics (solid boxes). (B) Phasic distension. Gastric compliance was the same for functional dyspeptics and healthy controls at each pressure tested. 
Table 3 Reporting of gastric sensations during sham gastric distension

\begin{tabular}{llll}
\hline & $\begin{array}{l}\text { Functional dyspepsia } \\
(n=23)\end{array}$ & $\begin{array}{l}\text { Organic dyspepsia } \\
(n=10)\end{array}$ & Controls $(n=15)$ \\
\hline Fullness & $12(52 \%)$ & $3(30 \%)$ & $6(40 \%)$ \\
Discomfort & $10(43 \%)$ & $3(30 \%)$ & $4(27 \%)$ \\
Pain & $10(43 \%)^{\star \star}$ & $3(30 \%)$ & $1(7 \%)$ \\
\hline
\end{tabular}

${ }^{\star \star} \mathrm{p}<0.01$ for functional dyspepsia $v$ controls.

disconnecting the air infusion device from the subject without his or her knowledge. During sham distension fullness was reported by $21 / 48$ subjects, discomfort by $17 / 48$, and pain by $14 / 48$ (table 3). Patients with FD, patients with organic disorders, and healthy volunteers were equally likely to report sensations of fullness and discomfort during sham distension. However, the patients with FD were more likely than normal controls but not organic dyspeptics to report pain during sham distension $(43 \%$ versus $7 \%$, $\mathrm{p}=0.01$ ). During sham distension, the "volume" at which sensations were reported was the same for FD, organic, and normal control subjects. Thresholds for fullness, discomfort, and pain reported during sham distension and actual distension were not different (fig 5).

There was no relation between reporting of sensations during sham distension and psychological symptoms, including anxiety and somatisation. However, statistical power for detecting an association is low due to the small number of subjects studied.

Patients who reported discomfort or pain during sham distension had lower thresholds for sensations of fullness, discomfort, and pain $(\mathrm{p}<0.02$ for each). When subjects with FD or organic dyspepsia were examined separately, sham response was associated with a reduced threshold for fullness in FD ( $F: p=0.03$; $D$ : $\mathrm{p}=0.08 ; \mathrm{P}: \mathrm{p}=0.18$ ) and for pain in organic dyspepsia ( $F: p=0.2 ; D: p=0.1 ; P: p=0.03$ ). Among healthy volunteers, those reporting sham had lower thresholds for discomfort and pain $(F: p=0.17 ; D: p=0.056 ; P: p=0.07)$.

\section{Discussion}

In the current study we have shown that even though patients with severe functional and organic dyspepsia have a similar prevalence of

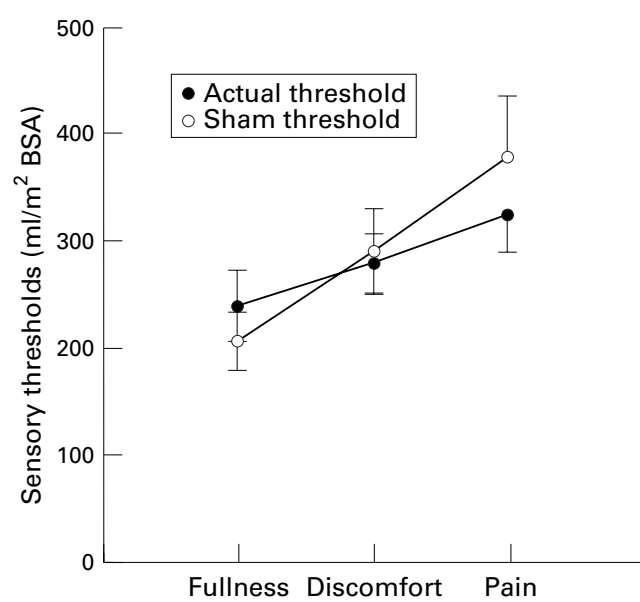

Figure 5 Perceptual thresholds during sham distension. Mean perceptual threshold volumes were measured for all subjects during actual gastric distension and during sham distension. dyspeptic symptoms, only those with functional disease show evidence of altered visceral perception in the form of lowered perception thresholds. Likewise, the prevalence of altered viscerosomatic referral during ramp gastric balloon distension was significantly more common in patients with FD.

In this study patients with FD and organic disease controls had similar clinical symptoms. There was no difference in meal associated exacerbation, reflux symptoms, or bloating between FD and organic disease. In contrast to conventional wisdom, we confirmed our earlier finding $\mathrm{s}^{15}$ that sleep disturbances are common in severe FD, and that weight loss is equally common in organic and functional dyspepsia. There was no difference in the prevalence of any of the subjective symptoms in those with and without hypersensitivity. In this population sample, symptoms from the upper gut therefore appear to be relatively non-specific. One could argue that the similarity of symptoms may result from the presence of FD in some or all of the patients with organic disease. While such an overlap of organic and functional symptoms may occur in some patients, it is unlikely to explain symptoms in the current study. In those $7 / 10$ patients with mucosal pathology, symptoms resolved promptly following appropriate therapy of the organic lesion. As recently suggested, ${ }^{16}$ attempts to classify patients with epigastric pain as reflux-like, ulcer-like, or dysmotility-like are therefore unlikely to separate patients into diagnostic or pathophysiological groups. The symptom of severe nausea, however, did occur more often in subjects with FD and was correlated with psychological distress, particularly anxiety. In this patient population seen at a tertiary referral centre, who rated their symptoms as severe, we found an equal prevalence of psychological symptomatology in the functional and organic group. However, the number of patients with psychological measurement data was small (19 FD and eight organic) and the resulting statistical power to detect a true difference is low. Nevertheless, in this study we believe it is unlikely that psychological symptoms were related to the hypersensitivity observed in the patients with FD. This finding supports the concept that psychological symptoms are a comorbid condition with FD and organic dyspepsia, which may play a role in a patient's decision to seek tertiary care.

In the current study we have applied balloon distension paradigms previously used to characterise alterations in rectal afferent function in patients with organic and functional intestinal disease. Since both rectum and stomach function as storage organs, and since a considerable overlap exists between symptoms of IBS and $\mathrm{FD},{ }^{17}$ we expected to see similar perception abnormalities in the two conditions. We have recently shown that IBS patients report the sensation of discomfort at lower degrees of rectal distension regardless of whether thresholds are expressed as pressure, volume, or wall tension. Furthermore, in the rectum this altered perception is limited to the sensation of discomfort, and is only observed during rapid, phasic distension, whereas thresholds during 
slow ramp distension are normal or even elevated in some patients. In contrast to IBS, we have found hypersensitivity in FD to both slow ramp and rapid phasic distension, and the reduced thresholds are found only in terms of volume of gastric distension, not pressure. In addition, the hypersensitivity is for both nonnoxious (fullness) and noxious sensations (discomfort and pain), as opposed to the finding in IBS patients. The observed difference may relate to at least three factors: differences in the pressure volume characteristics between the stomach and rectum; regional differences in afferent innervation; and regional differences in the psychophysics of visceral perception.

\section{PRESSURE VOLUME CHARACTERISTICS}

In the rectum, increasing balloon distension volumes are accompanied by increasing intrarectal pressures, reflected in a sigmoid pressure volume curve. In contrast, in the stomach, initial distension causes a steep rise in pressure and volume (slope phase), while further distension (generally above 300-400 ml) causes a slower rise in pressure with each volume increment (plateau phase). During ramp distension, fullness, discomfort, and pain are experienced in the plateau phase of the compliance curve, where large changes in volume are associated with no or very small changes in pressure. This may explain why pressure thresholds are not different between patient groups, or between sensations within a group. The gastric pressure volume relation has been evaluated by several investigators using either an infinitely compliant bag or a balloon as was used in this study. Other investigators ${ }^{8}$ have used elastic balloons for gastric distension and have also shown a two phase compliance curve with a plateau after $300-400 \mathrm{ml}$, similar to the findings we report using a balloon. Using a gastric balloon, Bradette et al reported reduced volume thresholds in $\mathrm{FD},{ }^{8}$ similar to the current findings. However, using a bag, Malagelada ${ }^{9}$ found the gastric-pressure volume relation to be linear (no plateau) at up to 600 $\mathrm{ml}$ of distension, and reported reduced pressure thresholds in patients with FD versus control subjects. The reason for these differences is unclear. Nevertheless, since compliant balloons were used both in the rectal studies of IBS patients and in the current study of gastric distension, the observed pressure volume differences between stomach and rectum are likely to represent true anatomical or physiological differences between the two organs.

AFFERENT INNERVATION

In the current study of FD, we provide evidence for alterations in visceral afferent pathways, mediating perception of gastric distension. Patients with FD had significantly lower pain and discomfort thresholds during ramp and phasic distension. Sensory thresholds measured during predictable volume ramp distensions may be influenced by response bias, as suggested by patient reports of sensations during sham distension. However, since the irregular sequence of the phasic distension steps was not known to the subjects, and the rate of isobaric volume expansion varies with the pressure step, and since visual and auditory cues were eliminated in our protocol, it would be extremely difficult for subjects to anticipate the degree of distension using temporal cues. In addition, half of the patients with FD showed altered viscerosomatic referral patterns, which are strongly suggestive of altered spinal or supraspinal processing of visceral afferent information. ${ }^{518}$

Both the rectum and stomach have a dual sympathetic and parasympathetic afferent innervation. It is generally assumed that vagal afferent fibres (parasympathetic) with mechanoreceptors located in the mucosa and muscle wall do not play a significant role in the mediation of visceral pain, even though they may be involved in pain modulation. ${ }^{19}$ Consistent with this concept is the observation that symptoms of dyspepsia are common in patients after truncal vagotomies, that perception thresholds for duodenal distension are lower in patients with truncal vagotomies, ${ }^{20}$ and that patients who have undergone gastric surgery including vagotomy for symptoms of intractable dyspepsia but without documented ulcer (presumably patients with FD) commonly experience an exacerbation of dyspepsia. ${ }^{21}$ As opposed to vagal afferents, splanchnic afferent pathways (sympathetic) are involved in the mediation of visceral pain. The receptive fields of these splanchnic afferents are thought to be located in the serosa and mesentery of the gut, with traction and torque the most appropriate stimuli to activate serosal tension receptors. ${ }^{22}$ Even though the relation between perception and the pressure volume relation in the stomach is consistent with balloon distension being encoded by a volume receptor, it is conceivable that the large volume required to elicit conscious perception in the stomach and the mobile mesentery of the stomach causes mesenteric traction during both rapid (phasic) and slow (ramp) distension. Conversely, with much smaller distension volumes used in the rectum, and the fact that the rectum is only partially invested by mesentery, traction on the rectosigmoid mesentery may occur primarily during rapid (phasic) distension.

Analogous to animal models of visceral afferent sensitisation, it has previously been suggested that mucosal irritation or inflammation may play a role in the development of visceral hyperalgesia. ${ }^{5}$ However, our results indicate that chronic inflammation in the gastrooesophageal region does not induce hypersensitivity since $7 / 10$ organic patients with normal sensitivity to gastric distension had inflammatory luminal lesions (gastric ulcer-one, duodenal ulcer-two, macroscopic oesophagitis-one, presumed microscopic oesophagitis - three), but none had hypersensitivity to gastric distension. In addition, chronic pain of organic aetiology in the gastrooesophageal region is not sufficient to alter perception of gastric distension. These findings are analogous to recent findings in patients with Crohn's disease, in whom chronic inflammation in the small bowel is not associated with rectal hypersensitivity. ${ }^{23}$ 
PSYCHOPHYSIOLOGY OF VISCERAL PERCEPTION In addition to showing alterations in afferent processing of gastric stimulation during phasic distension, we show evidence for a greater response bias in FD subjects during ramp distension. Even though it has received no attention in published rectal and gastric distension studies, response bias is a well known phenomenon in the psychophysiology of perception. ${ }^{24} \mathrm{~A}$ greater response bias during balloon distension of the oesophagus has previously been reported in patients with noncardiac chest pain. ${ }^{25}$ Response bias is indicated by the fact that all three study groups must have used cues other than sensory information encoded by gastric mechanoreceptors to judge the perceptual threshold of gastric sensations during sham ramp distension. Since subjects had no auditory or visual cues about the distending stimulus, but had experienced the time course of the ramp distension during the practice run, it is most likely that they used temporal cues (the memory of time course of the first distension) to rate perception thresholds. Even though mean perception thresholds during sham and actual ramp distension were not different in any of the study groups, a greater response bias in the FD group during ramp distension is suggested by our finding that patients with FD reported pain during sham distension more often than healthy controls, but not organic dyspeptics. Response bias in the current study was not related to psychological symptom severity, including somatisation. Even though response bias is traditionally viewed as a psychological phenomenon, recent results from functional brain imaging studies in healthy volunteers have clearly shown that the perceived intensity of a sham distension of the rectum correlates with the same brain regions as the actual distension. ${ }^{26}$ The current findings are consistent with our recent study in IBS patients, showing the presence of both visceral afferent hypersensitivity (determined during a distension paradigm minimising response bias, and manifested as lowered perception thresholds) and response bias (manifested in the earlier use of aversive terms to label visceral sensations). ${ }^{27}$ Results emphasise the need for careful control of response bias in future studies and clinical protocols.

In summary, we have found gastric balloon distension useful to characterise patients with chronic upper abdominal pain, since hypersensitivity was present in the majority of patients with functional disease and in our experience (using two control groups, organic dyspepsia and healthy volunteers) was highly specific for FD. Subjective symptoms, including psychological ones, were not useful in separating FD from organic dyspepsia with the exception of severe nausea, which was associated with FD. In patients with dyspepsia who complain primarily of nausea, a high index of suspicion for functional or psychiatric disease should be maintained. Abnormalities in perception of gastric stimuli are infrequent in patients with organic causes of dyspepsia, indicating that chronic pain, or chronic inflammation per se does not induce altered afferent function. Abnormalities in the perception of visceral stimuli are likely to underlie a number of functional gastrointestinal illnesses (IBS, FD, noncardiac chest pain) and categorising patients based on these physiological alterations may be more useful than the use of symptom criteria.

Supported in part by National Institute of Diabetes and Digestive and Kidney Diseases grant DK 40919 and by funds from the Veterans Administration.

1 Fiosne U, Kleveland PM, Waldum $\mathrm{H}$, et al. The clinical benefit of routine upper gastrointestinal endoscopy. Scand $\mathcal{F}$ Gastroenterol 1986;21:433-40.

2 Mansi C, Mela GS, Savarino V, et al. Open access endoscopy: a large scale analysis of its use in dyspeptic patients. $\mathscr{f}$ Clin Gastroenterol 1993;16:149-54.

3 Drossman DA, Li A, Adruzzi E, et al. US household survey of functional gastrointestinal disorders. Dig Dis Sci 1995;9: of functional

4 Mayer EA, Raybould HE. Role of visceral afferent Mayer EA, Raybould $\mathrm{HE}$. Role of visceral afferent
mechanisms in functional bowel disorders. Gastroenterology 1990;99:1688-704.

5 Mayer EA, Gebhart GF. Basic and clinical aspects of visceral hyperalgesia. Gastroenterology 1994;107:271-93.

6 Lembo T, Munakata J, Mertz H, et al. Evidence for the hypersensitivity of lumbar splanchnic afferents in irritable bowel syndrome. Gastroenterology 1994;107:1686-96.

7 Mertz H, Naliboff B, Munakata J, et al. Altered rectal perception is a biological marker of patients with the irrita-
ble bowel syndrome. Gastroenterology $1995 ; \mathbf{1 0 9} 40-52$.

8 Bradette M, Pare P, Douville P, et al. Visceral perception in health and functional dyspepsia. Crossover study of gastric distension with placebo and domperidone. Dig Dis Sci
1991;36:52-8.

9 Malagelada, JR. Altered visceral sensation in functional dyspepsia and related syndromes. In: Mayer EA, Raybould HE, eds. Basic and clinical aspects of chronic abdominal pain.

Amsterd Basic and climical aspects of

10 Mertz H, Sytnik B, Galen S, et al. Evidence for altered spinal processing of gastric afferent information in non-ulce dyspepsia [abstract]. Gastroenterology 1993;104:A551

11 Talley NJ, Phillips SF, Wiltgen CM, et al. Assessment of functional gastrointestinal disease: the bowel disease questionnaire. Mayo Clin Proc 1990;65:1456-79.

12 Derogatis LR. SCL-90R. Administration, scoring and procedures manual-II. Towson, Maryland, 1983.

13 Plourde V, Lembo T, Shui Z, et al. Effects of the somatostatin analogue octreotide on rectal afferent nerves in humans. Am f Physiol 1993;265:G742-5.

14 Mertz H, Walsh JH, Sytnik B, et al. The effect of octreotide on human gastric compliance and sensory perception. Neurogastroenterol Motil 1995;7:175-95.

15 David D, Mertz H, Fefer L, et al. Sleep and duodenal motor activity in patients with severe non-ulcer dyspepsia. Gut 1994;35:916-25.

16 Talley NJ, Zinsmeister AR, Melton III LJ. Irritable bowel syndrome (IBS) and symptom subgroups: prevalence, onset, and risk factors [abstract]. Gastroenterology 1994; 106:A577.

17 Talley NJ. Spectrum of chronic dyspepsia in the presence of the irritable bowel syndrome. Scand $\mathcal{f}$ Gastroenterol 1991;182:7-10.

18 Apkarian AV, Brueggemann J, Shi T, et al. A thalamic model for true and referred visceral pain. In: Gebhart GF, ed. Visceral pain. Seattle: IASP Press, 1995:217-60

19 Sengupta JN, Gebhart GF. Gastrointestinal afferent fibers and sensation. In: Johnson LR, ed. Physiology of the gastrointestinal tract. New York: Raven Press, 1994.

20 Holtmann G, Neufang-Hueber J, Fischer H, et al. Influence of the vagal nerve on intestinal mechanosensory function and intestino-intestinal reflexes in humans [abstract]. Gastroenterology 1996;110:A680.

21 Johnstone FR, Holubitsky IB, Debas HT. Post-gastrectomy problems in patients with personality defects: the "albaproblems in patients with personality defects: the

22 Jaenig W, Morrison JFB. Functional properties of spinal visceral afferents supplying abdominal and pelvic organs, with special emphasis on visceral nociception. Prog Brain Res special emp

23 Bernstein CN, Robert ME, Kodner A, et al. Is there an irritable bowel syndrome (IBS) component in patients with ileal Crohn's disease (CD)? [abstract]. Gastroenterology 1993;104:A477.

24 Gracely RH. Pain psychophysics. In: Chapman CR, Loeser $\mathrm{D}$, eds. Advances in pain research and therapy: issues in pain measurement. New York: Raven Press, 1995:211-29.

25 Bradley LA, Richter JE, Scarinci IC, et al. Mechanisms of altered pain perception in non-cardiac chest pain patients [abstract]. Gastroenterology 1993;104:A482.

26 Silverman DHS, Munakata J, Hoh CK, et al. Regional cerebral activity in normal and pathologic perception of brion of pain: an O-15-water PET study of healthy subjects and 759 .

Naliboff B, Munakata J, Gracely R, et al. IBS patients with low rectal discomfort thresholds show normal stimulusresponse functions for rectal sensations [abstract]. Gastroenterology 1995;108:A656. 